\title{
Searching for the Nearest Extragalactic Binary Black Hole: A Spectroscopic Study of NGC 4736
}

\author{
Annika Gustafsson, Physics and Math*
}

\begin{abstract}
Maoz et al. $(1995,1996)$ concluded that the nearby galaxy NGC 4736 is in the late stages of a merger event. After further investigation, Maoz et al. (2005) observed variability in the nuclear region of NGC 4736, revealing a second unknown source of radiation in the nucleus. Since merging systems are an ideal location to search for binary black holes $(\mathrm{BBH})$, we hypothesized that the second source could be a second black hole, making this a potential BBH system. Observational evidence for the existence of BBH remains sparse, even though $\mathrm{BBH}$ are predicted by many theories. To date, only NGC 6240 (Komossa et al., 2003) and Arp 299 (Ballo et al., 2004) have been discovered as merging galaxies with two active galactic nuclei (AGN). In 2008, NGC 4736 was observed with the Gemini-North telescope. We can classify the nature of the unknown source by looking at the optical line ratios following Ho et al. (1997). High signal-tonoise spectra of the unknown source displayed strong emission of [SII] and [NII], but extremely weak [OIII] emission. The unknown source has a calculated $[\mathrm{NII}] /[\mathrm{H} \alpha]$ ratio of 1.37 and an upper limit of 0.6 for the $[\mathrm{OIII}] /[\mathrm{H} \beta]$ ratio. Placing the unknown source on the BPT-NII diagram (Baldwin et al., 1981), we tentatively conclude that it is a second black hole potentially making NGC 4736 the nearest BBH system. The result will enable future observations of a low-luminosity system in extremely late stages of merging, which will be a significant step forward in validating models of galaxy mergers and AGN activity that further our understanding of galaxy formation and evolution.
\end{abstract}

\section{INTRODUCTION}

There are two known astronomical binary black hole systems where both black holes in the nucleus of a single galaxy exhibit active galactic nuclei (AGN) behavior. The first binary black hole (BBH) system was discovered in galaxy NGC 6240, approximately 40 million light years away, by Komossa et al. in 2003 when imaging from the Chandra X-ray Observatory revealed two X-ray sources in the nuclear region of the galaxy. The second, and only other candidate for a BBH system Arp 299, approximately 130 million light years away, was discovered in 2004. NGC 4736 was added to the list of candidates when Maoz et al. (2005) made UV wavelength observations and discovered two sources of UV radiation in its nucleus. Anomalies in the spectrum of the galaxy led Maoz et al. (2005) to hypothesize that the galaxy is in the late stages of a merger, and thus, a likely place to look for a $\mathrm{BBH}$ system. If confirmed as a $\mathrm{BBH}$, this system would represent a much more advanced stage of merging than the previously discovered $\mathrm{BBH}$

\footnotetext{
*Annika Gustafsson, a native of Santa Cruz, California, is a senior physics and mathematics major and business minor. She is also a member of the Robert D. Clark Honors College. Annika's academic interests have always lied in astronomy and physics. She plans to receive her doctoral degree in Planetary Science with the intentions of pursuing a career in observational research.
} 
systems. Hence, the discovery would be extremely important in helping validate theoretical predictions by providing the opportunity to study an older, and presumably more evolved system. Additionally, at a distance of only 16 million light years, NGC 4736 is much closer than the other two galaxies, which offers the ability to study such a system in greater detail.

While $\mathrm{BBH}$ are hypothesized to be common due to the frequency of merging galaxies, there is still little observational evidence to support their existence. However, many theories and computer models support the existence of BBH systems because they can help explain many predicted astrophysical phenomena. One obvious place to look for these systems is in merging galaxies. Collisions, or mergers, occur when galaxies become too close and their gravitational forces pull them together, which results in them joining, or merging. In 2005, evidence from the Hubble Space Telescope led astronomers to predict that black holes and galaxy formation go hand in hand. As a result, it is predicted that every galaxy contains a black hole in its nucleus. Some of these black holes are active, while others are completely dormant. An active galactic nucleus (AGN) is a region at the center of a galaxy, most often with a black hole, which produces enormous luminosities and intensities, sometimes as much as an entire galaxy. The high luminosities result in distinctive spectra that make these systems relatively easy to detect as they are quite different from the spectra of most stars and galaxies whose spectral lines are generally weak and predominately in absorption. The mechanism that causes a black hole to turn its activity on and off is still largely unknown; therefore, studying black holes at extremely low luminosities is important in determining the links between a very luminous, or active, black hole and a quiescent black hole.

When two galaxies merge, the resulting merged galaxy will likely have two black holes in its nucleus, one from each host galaxy, until the larger, or more massive black hole, consumes the smaller. Theories that rely on the existence of BBH systems include BBH triggering star formation and initiating the formation of dusty AGN tori. They can also explain observations as diverse as helically distorted radio jets and quasi-periodic variations in the light curves of some BL Lac objects.

\section{PROPOSAL}

We proposed to analyze extant optical spectroscopy data to determine the exact nature of this unknown second source in the nucleus of NGC 4736. I completed this project under the assistance of my research group at University of Oregon, which consisted of two fellow undergraduate physics majors, my advisor Dr. Scott Fisher, and Dr. Rachel Mason.

Dr. Rachel Mason, Assistant Astronomer at Gemini Observatory in Hilo, Hawaii recognized the importance of the discovery of the second unknown source by Maoz et al. (2005), and submitted a proposal for telescope time at Gemini-North to observe both the known black hole and the second source with the intention of collecting data that would shed light on the nature of the unknown source. Dr. Mason invited me to reduce and analyze the data for this project. 


\section{HYPOTHESIS}

NGC 4736 has been observed in the optical (Taniguchi et al., 1996), UV, and X-ray wavelengths of light (Cui et al., 1997, Roberts et al., 1999 \& Eracleous et al, 2002). The observations made in the optical were performed using a 2 " slit on a small telescope (Figure 1). Due to the lack of spatial resolution, it was impossible to differentiate the two separate sources in the nucleus. Therefore, the much more luminous source dominated the spectrum and the signatures of the unknown source were lost. Our observations from the 8-meter Gemini-North Telescope using a 0.75 " slit provides the resolution needed to spatially resolve the entire nucleus at the primary and secondary source.

There is strong evidence to support our hypothesis that the second source is in fact a lowluminosity AGN. NGC 4736 has already been classified as an AGN due to activity of the primary source in the nucleus. Thus, discovering the nature of the second source to be a black hole would be important as the galaxy would then be the third known BBH system.

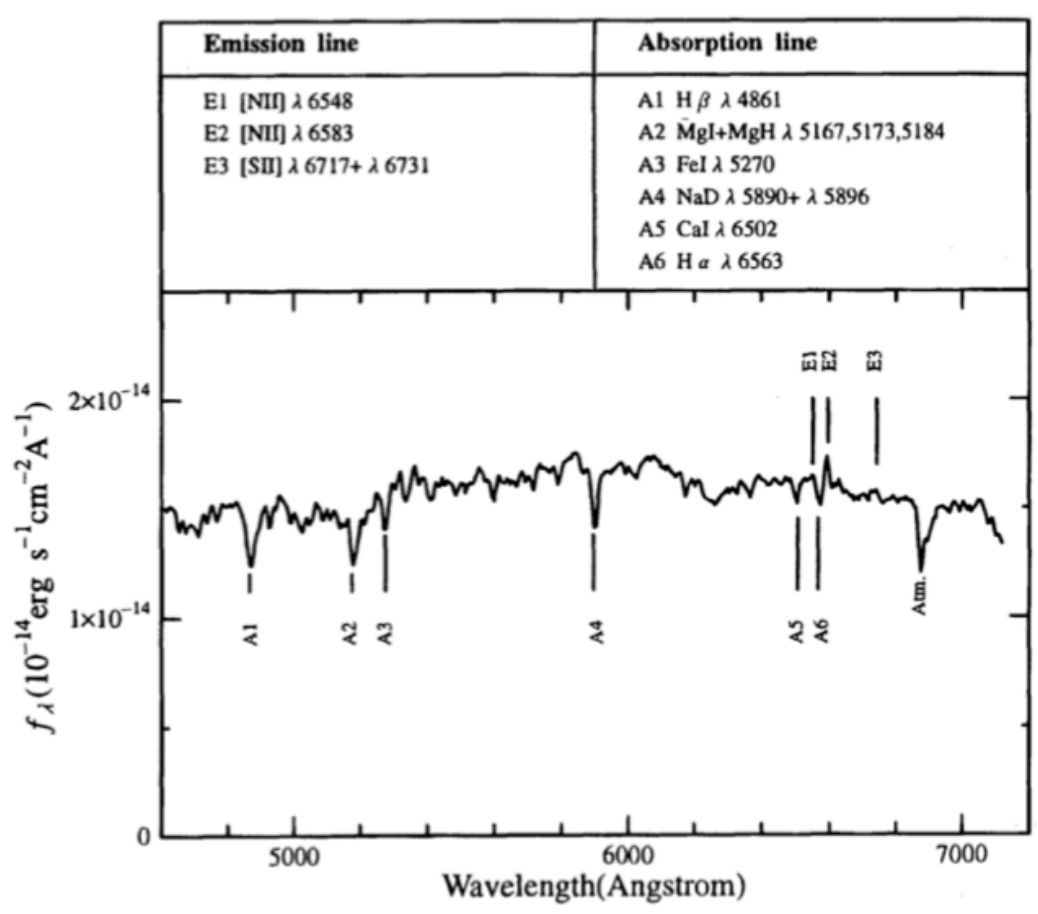

Figure 1: NGC 4736 spectrum from Taniguchi et al. (1996). Notable diagnostic lines are emission in $[\mathrm{NII}]$ and absorption in $[\mathrm{H} \beta]$ and $[\mathrm{H} \alpha]$.

Diagnostic diagrams, like the one created by Baldwin, Phillips \& Terlevich (Baldwin et al., 1981) referred to as the BPT-NII Diagram (Figure 2), help to distinguish the spectral differences between various types of objects including AGN and star forming regions. The Balmer series lines of hydrogen appear in the spectrum of many astronomical objects due to the abundance of hydrogen in space. It is known that $\mathrm{H} \alpha$ and $\mathrm{H} \beta$, two of the Balmer lines, are present throughout the spectrum of all AGN, from quiescent black holes to extremely active black holes. The primary difference in the spectra of AGN and non-AGN sources are the prevalence of high 
excitation emission lines from NII, SII, and OIII. Objects which have a low [NII]/[Ha] and $[\mathrm{OIII}] /[\mathrm{H} \beta]$ ratio are most likely star forming regions, also called HII regions. Objects with a higher $[\mathrm{NII}] /[\mathrm{H \alpha}]$ and $[\mathrm{OIII}] /[\mathrm{H} \beta]$ ratio are classified as AGNs, falling into the Seyfert or LINER categories. The transition line displayed on the BPT plot (Figure 2) separates the AGN objects on the right from the non-AGN objects on the left.

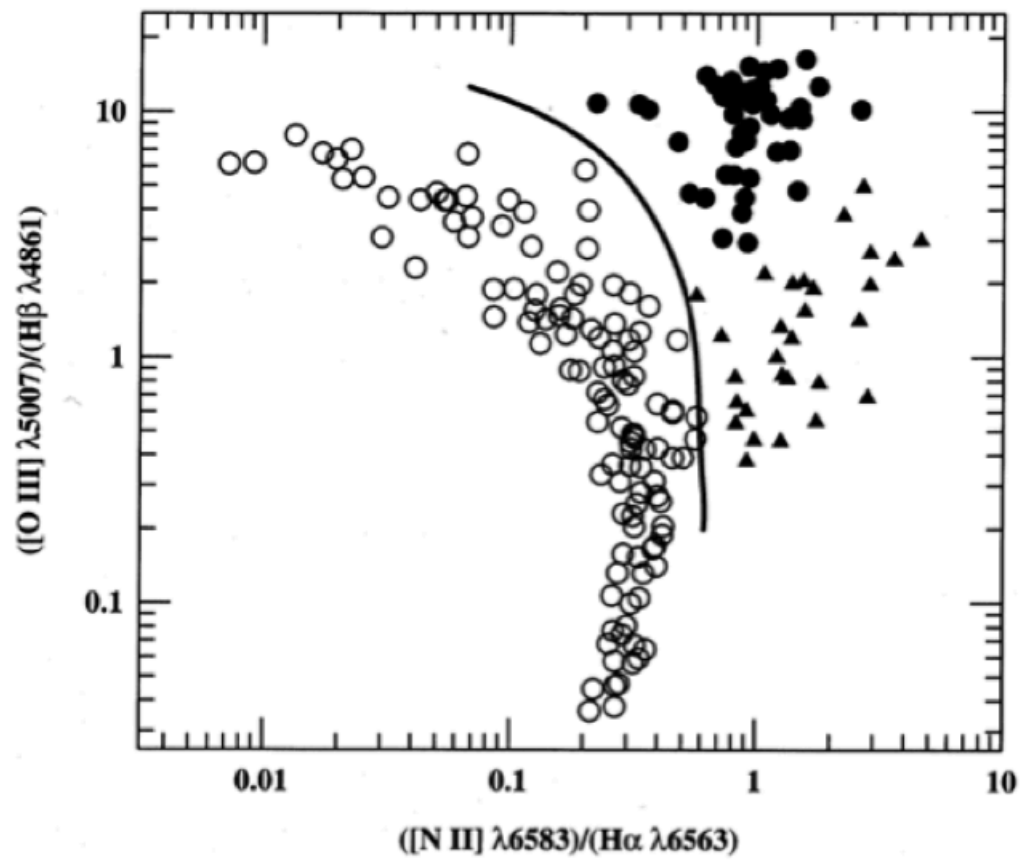

Figure 2: A BPT Diagram helps to distinguish between the different categories of objects that contribute to AGN activity. The open circles represent HII regions, the closed circles represent Seyferts, and the triangles represent LINERs. The axes of the plot, $[\mathrm{OIII}] /[\mathrm{H} \alpha]$ and $[\mathrm{NII}] /[\mathrm{H} \beta]$, contain typical emission line elements of these objects (Baldwin, Phillips, $\&$ Terlevich, 1981).

There are two types of Seyfert galaxies. The classification as a Seyfert 1 or Seyfert 2 depends on the orientation of the galaxy. A Seyfert 1 galaxy is oriented "face on". As such, an observer can view only the black hole jet coming straight out because the galaxy itself blocks the second jet. A Seyfert 2 galaxy is oriented "edge on" allowing the observer to see both the jet above and below the disk of the galaxy. NGC 4736 is a Seyfert 1. This is advantageous because it allows us to see directly down into the nucleus of the galaxy and spatially resolve the two nuclear sources. There are many emission lines we expect to see in a Seyfert 1 galaxy including [Ha], [NII], [SII], [HeI], and many others (Figure 3). 


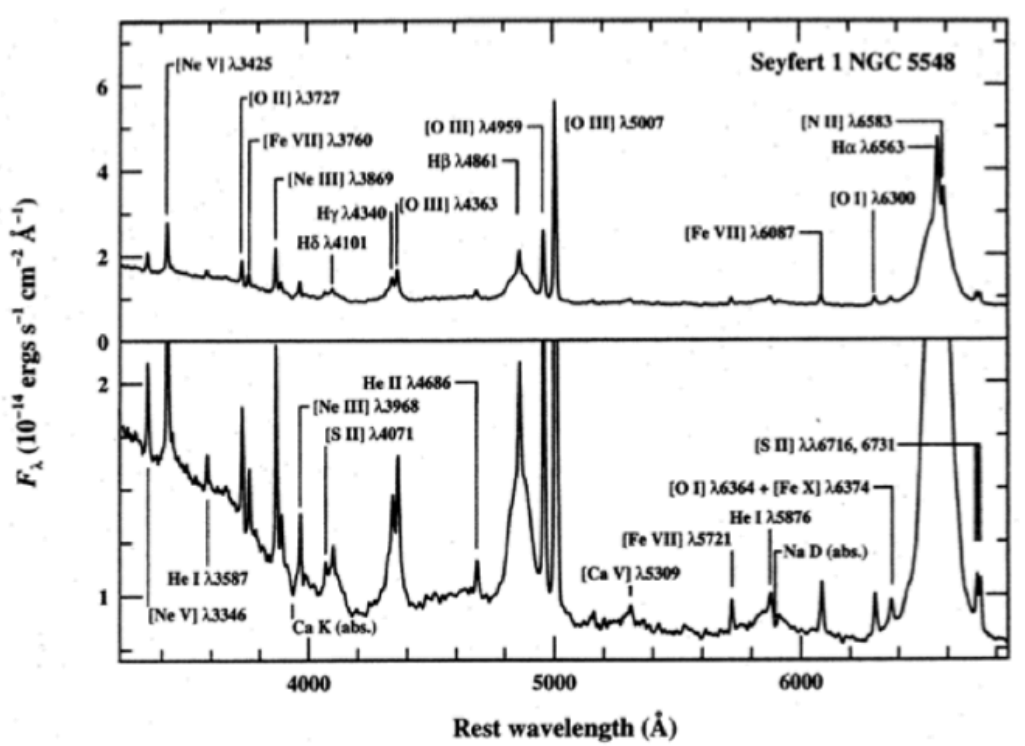

Figure 3: Expected spectra for a Seyfert I galaxy. A Seyfert 1 galaxy, oriented face on, has many characteristic emission lines including $[\mathrm{H} \alpha],[\mathrm{NII}],[\mathrm{SII}]$, and $[\mathrm{OIII}]$ (Peterson, 1997).

A further sub classification of Seyfert galaxies is a special class of object called a Low Ionization Nuclear Emission Region (LINER), a low energy AGN. The spectra of LINERs typically include emission lines from weakly ionized or neutral atoms, such as [O], [OI], [NI], and [SI]. Conversely, the spectral line emission from strongly ionized atoms, such as [OII], [NeII], and [HeI] are relatively weak. The primary source in the nucleus of NGC 4736 has been identified as having LINER behavior (example in Figure 4), but has also been included in catalogs of Transition objects (Constantin \& Seth, 2011).

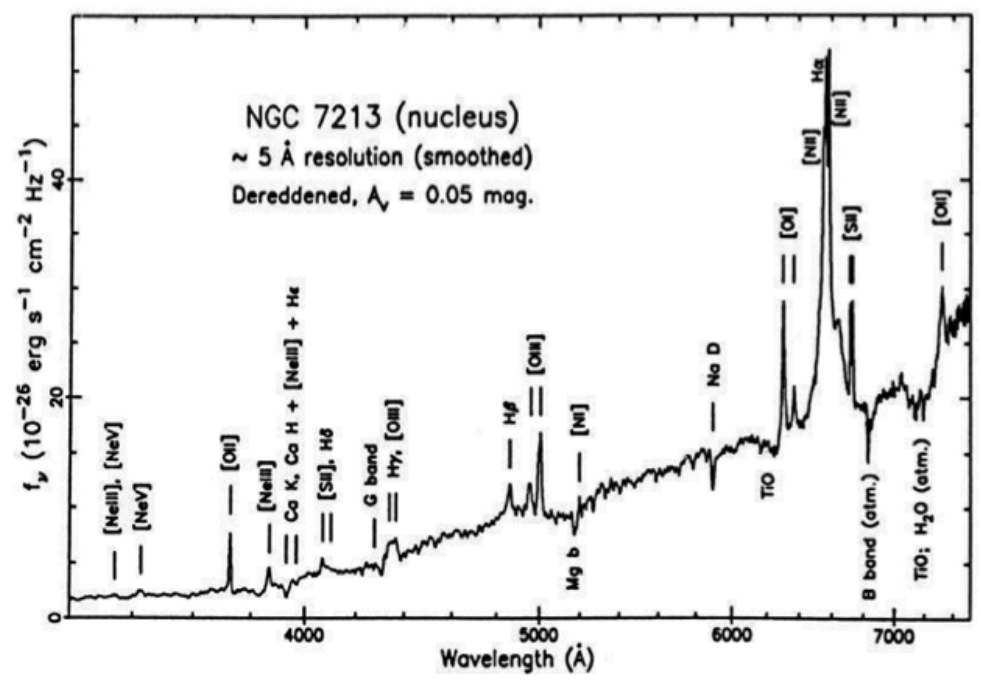

Figure 4: Expected spectra for a LINER galaxy. We expect much weaker emission lines from $[\mathrm{OII}]$, [NeII], and [HeI], and emission of weakly ionized atoms like [O], [OI], [HeI], and [SI] (Filippenko \& Halpern 1986). 
In contrast to the signatures of Seyfert and LINER AGN, young, hot stars mainly power the emission line activity of HII regions. Approximately 50\% of the strong line emitter objects fall into this category. Spectra of HII regions will have prominent strong emission lines for hydrogen and are typically associated with the presence of giant molecular clouds, like the Orion Nebula.

The most valuable research done on NGC 4736 thus far has been X-ray observations. Roberts et al. (1999) and Eracleous et al. (2002) showed evidence of a second source consistent with Seyfert spectra. Observations by Fernandes et al. (2004) ruled out evidence of luminous evolved stars. Together, these observations support AGN activity from a black hole and not an HII region. As a result, we can look at the optical spectra to definitively classify the second source as a black hole.

\section{METHODS}

Dr. Mason defined the technical methods required for obtaining the optical spectroscopic data of NGC 4736 (Appendix A.2). She proposed the use of the Gemini Multi-Object Spectrograph (GMOS) with long slit spectroscopy on the Gemini North telescope, located in Hilo, HI. The data was successfully taken in January of 2008 and the raw data was provided to my research group at University of Oregon in Spring 2013 to be reduced and analyzed (Table 1). Four data files of NGC 4736 were taken along with the necessary calibration files.

\section{Table 1: Summary of Observations}

\begin{tabular}{cccccc}
\hline \hline Type & Filter & Exposure (s) & Grating & Prefix & File \\
\hline NGC 4736 & open & $60.5 \mathrm{~s}$ & B600 & N20080109S & $0220-0222$ \\
NGC 4736 & open & $60.5 \mathrm{~s}$ & B600 & N20080109S & $0224-0225$ \\
NGC 4736 & open & $60.5 \mathrm{~s}$ & B600 & N20080109S & $0226-0227$ \\
\hline Flat & open & $4.0 \mathrm{~s}$ & B600 & N20080109S & 0223 \\
Flat & open & $4.0 \mathrm{~s}$ & B600 & N20080109S & 0228 \\
\hline ARC & open & $25.0 \mathrm{~s}$ & B600 & N20070627S & 0547 \\
ARC & open & $25.0 \mathrm{~s}$ & B600 & N20080109S & 0262 \\
\hline Bias & N/A & $0.0 \mathrm{~s}$ & N/A & N20080103S & 0175 \\
\hline Twilight Flat & N/A & $300.0 \mathrm{~s}$ & N/A & N20070627S & $0468-0469$ \\
Twilight Flat & N/A & $10.0 \mathrm{~s}$ & N/A & N20070627S & $0471-0472$ \\
\hline Std Star Aq. & N/A & $4.0 \mathrm{~s}$ & N/A & N20070627S & $0460-0462$ \\
Std Star Obs. & N/A & $120.0 \mathrm{~s}$ & N/A & N20070627S & 0463 \\
Std Star Flat & open & $4.0 \mathrm{~s}$ & B600 & N20070627S & 0464 \\
\hline
\end{tabular}

Table 1: Observation log for raw calibration and object data files. 


\subsection{DATA FILES}

The GMOS instrument was placed on the telescope in July of 2001. The spectrometer contains three separate charged coupled device (CCD) chips with a gap between each chip, 2.8 $\operatorname{arcsec}(39$ pixels) and an overscan region of 32 pixels (Figure 5). The data is also outputted with short wavelengths on the right and long wavelengths on the left.
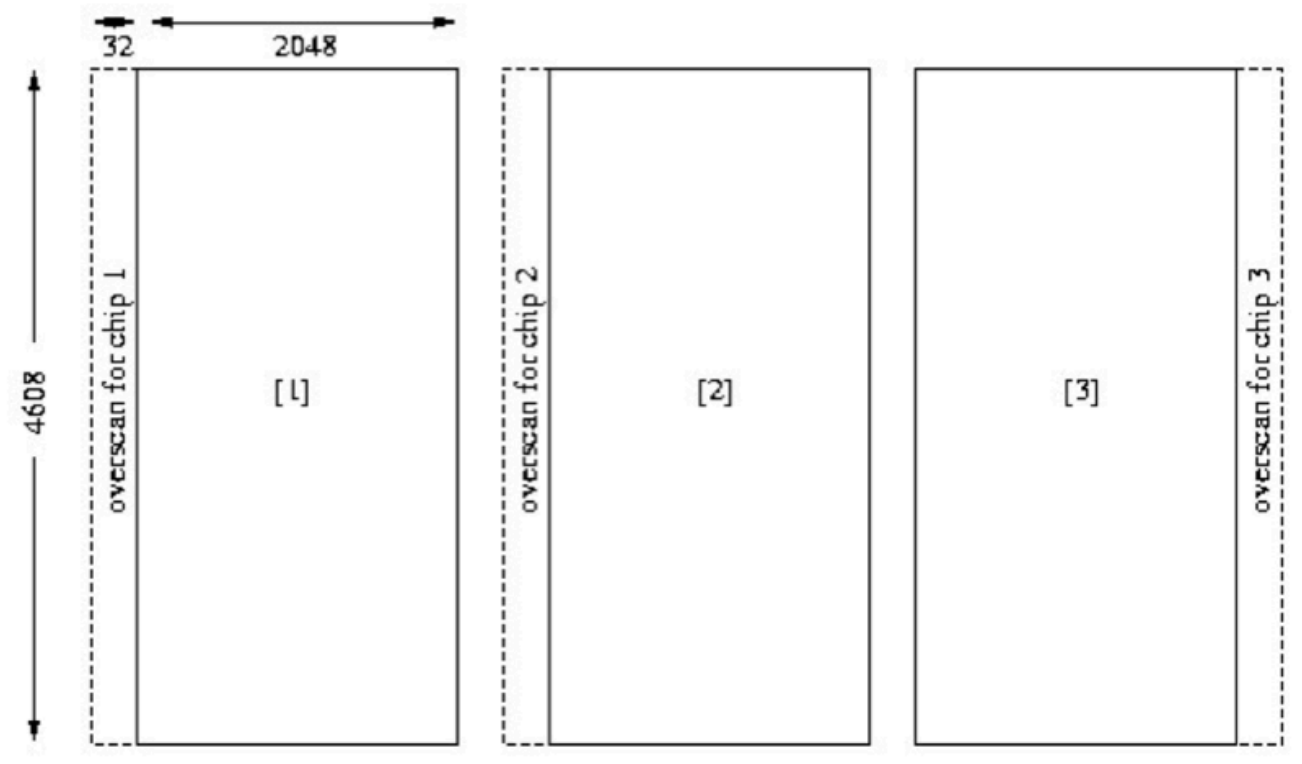

Figure 5: The GMOS instrument contains three separate CCD chips for detection, each of size $2048 \times 4608$ pixels with a 39 pixel gap in between each chip.

There are four main types of observations that are produced when using a CCD for optical spectroscopy: bias images, flat fields, object data, and an arc file. The bias images are used to calculate the zero noise level of the CCD. This is a pixel intensity offset that is inherent to the device, so once calculated, it gets subtracted off all images. A bias offset is used to avoid getting negative intensities in the CCD readout when taking data. There are two ways to evaluate the bias offset--bias images and the overscan region. Bias frames are zero second exposures taken with the shutter closed to have data of unexposed pixels on the readout. The overscan region is a set of rows and columns that are added to each data image. These are not physical rows or columns, but instead pseudo rows and pseudo columns created by scanning additional cycles to the readout. The bias frames are usually more useful than overscan regions because they represent a 2D bias image where you can subtract the bias pixel by pixel off each image, while the overscan is a $1 \mathrm{D}$ vector.

Flat fields are used in a correction process of the detector response. CCDs are not perfect imaging devices. Within the $\mathrm{CCD}$, each pixel responds differently to the light coming in, so it has a different quantum efficiency value when compared to its neighboring pixels. The purpose of a flat field image is to correct for this inconsistency. The goal is to observe a flat field that consists of uniform illumination of every pixel by a light source that has an identical response to that of 
the object data. By dividing by a normalized flat field, the pixel-to-pixel variations are removed. There are two types of flat fields - dome flats and twilight flats. Dome flats are taken using a uniformly illuminated screen on the inside wall of the dome while twilight flats are taken on a blank piece of sky. While flat fields are primarily used to correct for the pixel-to-pixel variations, they are also used to correct for dust donuts that appear on the image frames as a result of dust on the filters or other optical surfaces. Twilight flats were used for the data reduction in this research project.

The arc is the wavelength calibration file that allows for the calibration of the $\mathrm{x}$-axis scale on plots from pixels to wavelength. GMOS-N uses a standard CuAr lamp for wavelength calibration because the spectrum contains a large number of strong emission features between $4000 \AA$ and $7000 \AA$.

The object data for this project consists of four images taken with a 0.75 " long slit over both the primary and second source of the galaxy using the B600 grating which disperses the light at $0.045 \mathrm{~nm} /$ pixel. All of the data files were reduced such that all atmospheric and machine effects were removed. This reduction process produces the final, clean version of the data that we used in our interpretation.

\subsection{DATA REDUCTION}

The programming languages Interactive Data Language (IDL) and Image Reduction and Analysis Facility (IRAF) were used to reduce and analyze the GMOS data of NGC 4736. The method for reducing data in IDL is a much more interactive process, while IRAF has many procedures pre-written for convenience. Both software programs require the same reduction steps. The steps include bias subtraction, trimming, overscan correction, flat field correction, and wavelength calibration. These standard reduction steps were performed on all data files in both languages to verify that the data was correctly reduced. A detailed description of the reduction is outlined in Appendix $\mathrm{C}$.

Gemini Staff Scientist Dr. Kathleen Labrie performed the reduction process in parallel. Every step until the spectra extraction step was consistent between our output files and Dr. Labrie's output files. Our outputted spectra had a flux roughly three times greater than Dr. Labrie's outputted spectra (Figure 6). The mean of the ratio of our extraction to Dr. Labrie's was $2.93(\sigma=0.099)$. Since the absolute calibration of the data is not critical, we chose to proceed forward with Dr. Labrie's outputted data. In the end, this factor of three should not have a significant impact on the data results because we are looking at the relative strengths of the emission and absorption lines ratios and are not interested in the flux value. 


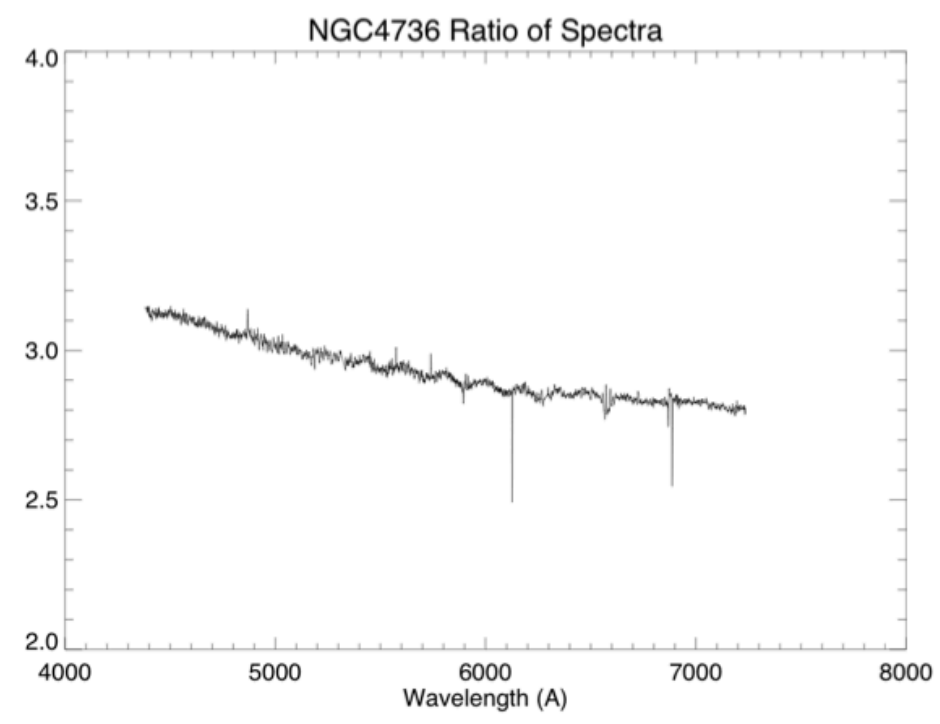

Figure 6: Ratio of Fishergroup extraction to Dr. Labrie's extraction of the nucleus of NGC 4736. ( $\mu=2.93$ and $\sigma=0.0992)$.

\subsection{DATA ANALYSIS}

\subsubsection{SPECTRA EXTRACTION}

The goal was to extract spectra over the known black hole referred to as the primary source, over the unknown source located at 2.5" north of the primary source, and over a baseline, or control, located 2.5" south of the primary source. By placing a range of spectra on the same plot, we plan to compare optical spectral features spatially across the nucleus to determine the nature of the second source. Based on our hypothesis that the second source is a second black hole, the most useful diagnostic emission and absorption lines with given wavelengths are likely to be

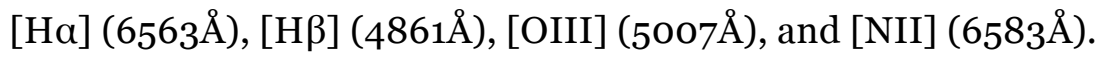

These diagnostic lines will determine the placement of the second source on the BPT diagram and help to definitively prove that NGC 4736 is in fact a BBH system in late stages of merging, allowing astrophysicists to begin validating theoretical predictions about both mergers and AGN.

We performed the spectra extraction in IDL. We extracted spatially across the nucleus of NGC 4736 using an aperture of 1 " from 5 " North of the nucleus to 5 " South of the nucleus about every 1 ". The Gemini data has a spatial resolution in y of 0.1454 "/pixel. We chose to use a 1 " aperture to match the seeing conditions at the time of the data collection. For a 1 " aperture, we used 7 pixels in $y$. Table 2 shows the specific extraction rows for our individual spectra from the image. 
Table 2: NGC 4736 Spectra Extraction

\begin{tabular}{cc}
\hline \hline Location & [Start Pixel:End Pixel] \\
\hline $5^{\prime \prime}$ North & $1380: 1386$ \\
$3.75^{\prime \prime}$ North & $1388: 1394$ \\
$2.5^{\prime \prime}$ North & $1397: 1403$ \\
$1.25^{\prime \prime}$ North & $1405: 1411$ \\
\hline Nucleus & $1414: 1420$ \\
\hline $1.25^{\prime \prime}$ South & $1423: 1429$ \\
$2.5^{\prime \prime}$ South & $1431: 1437$ \\
$3.75^{\prime \prime}$ South & $1440: 1446$ \\
$5^{\prime \prime}$ South & $1448: 1454$ \\
\hline
\end{tabular}

Table 2: 1" (7 pixel) extractions were taken across the nucleus of NGC 4736 in order to compare composition of the unknown source with the stellar population of the galaxy.

\subsubsection{LINE STRENGTHS}

Once we had our nine extractions across the nucleus, we began to classify the second source. To do this, we calculated line strengths for the diagnostic emission and absorption lines to search for changes between the primary and secondary source. We were interested in the diagnostic lines of the BPT-NII diagram- [NII], [Ha], [OIII], \& [Hß]. It is common that a continuum will be fit to the data in order to offset the shape of the spectra. Instead, we made the assumption, following advice from Gemini Staff Scientist Dr. Inger Jorgenson, that each deviation from the mean in the spectra was an emission or absorption line and that there is in fact no continuum for data of this quality. As a result, we did not fit a stellar population model to the entire spectra and instead isolated local continuum regions near the lines of interest to use as our baselines. We fit the baseline, or local value, to the line by averaging the continuum immediately to the right and left of the spectral feature. We subtracted this baseline and integrated under the curve using the Riemann Sum method with bin width of 1 pixel to calculate the strength of the line. If the line of interest was an absorption line, we took the absolute value before integrating so that all line strength values were positive.

\section{RESULTS}

1. The extraction of the nucleus of NGC 4736 (Figure 7) is consistent with what was seen in Taniguchi et al., 1996 (Figure 1). We see emission lines for [NII] and both [SII] lines. We also see emission within a larger absorption for $[\mathrm{H \alpha}]$ and absorption lines for $[\mathrm{H} \beta]$, [HeII], and [OI]. Along with [OIII] and [HeI] which are too weak to see clearly in the plot, these are the key diagnostic lines for identifying AGN. 
2. There is visual evidence to support that the emission and absorption lines are changing spatially across the nucleus of NGC 4736 from 5 " North to 5 " South (Figure 8). We plotted the region around $[\mathrm{Ha}]$ at the unknown source (2.5” North), the primary source, and at the control location (2.5" South) (Figure 9). The plots show clear evidence that $[\mathrm{Ha}]$ is in fact changing across the nucleus, suggesting that something is present in the North at the location of the unknown source that is not in the South.

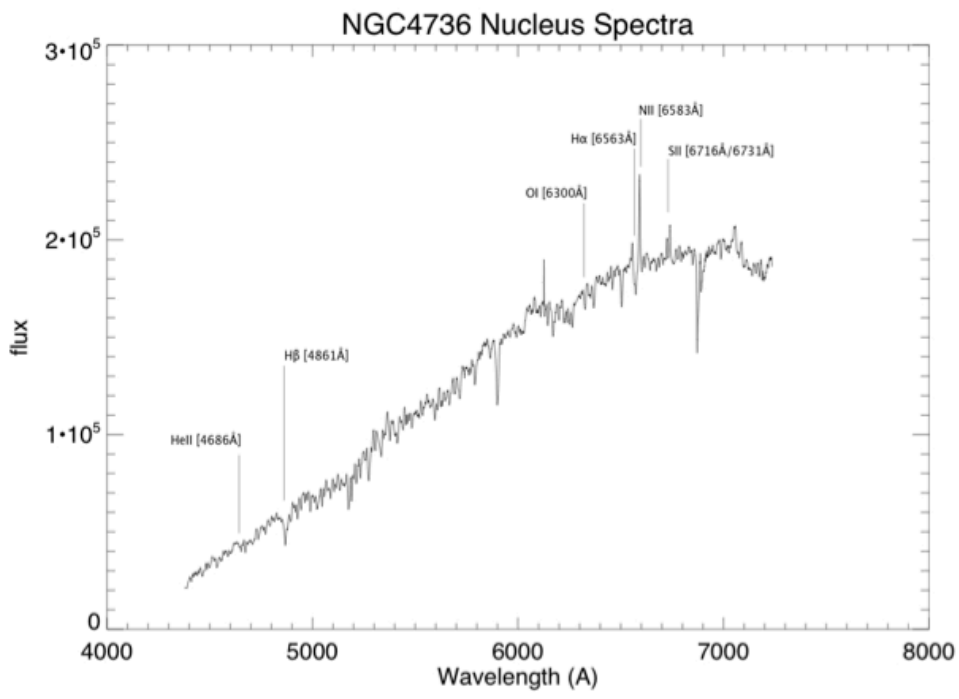

Figure 7: NGC 4736 spectrum of the primary source using a $1^{\prime \prime}$ aperture. The emission and absorption lines are consistent with what was seen in Taniguchi et al., 1996 using a much smaller telescope.

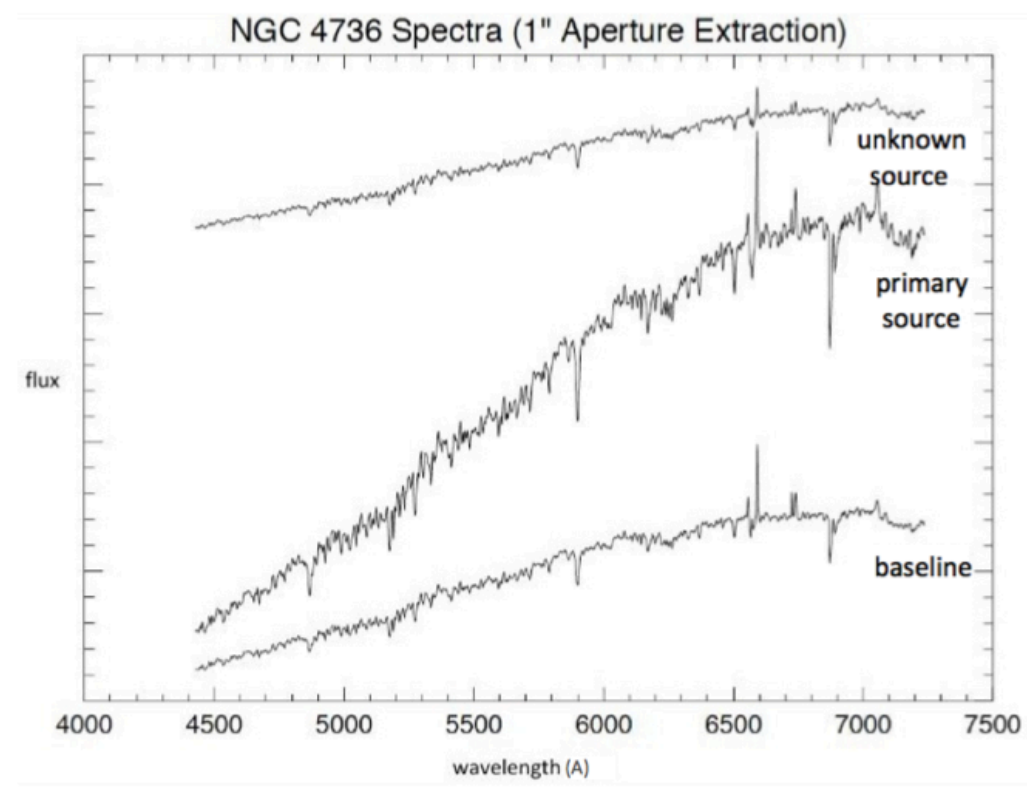

Figure 8: NGC 4736 spectra north $2.5^{\prime \prime}$ and south $2.5^{\prime \prime}$ of the primary source. These are the three key spectra that we will use to determine the nature of the unknown source. 


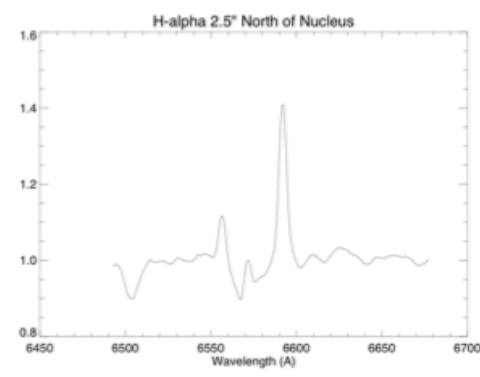

(a)

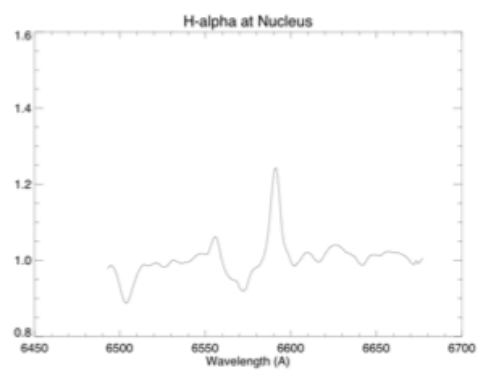

(b)

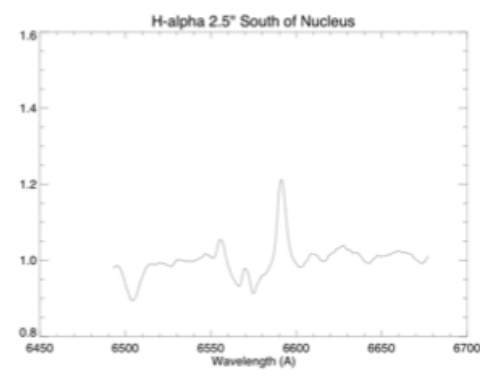

(c)

Figure 9: $[\mathrm{H} \alpha](6563 \AA)$ emission within absorption as it changes across the nucleus of NGC 4736.

3. A different spectral shape is observed at the long wavelength end of the spectrum, which implies that the unknown source is bluer, and therefore presumably hotter, than the background, or local environment (Figure 10).

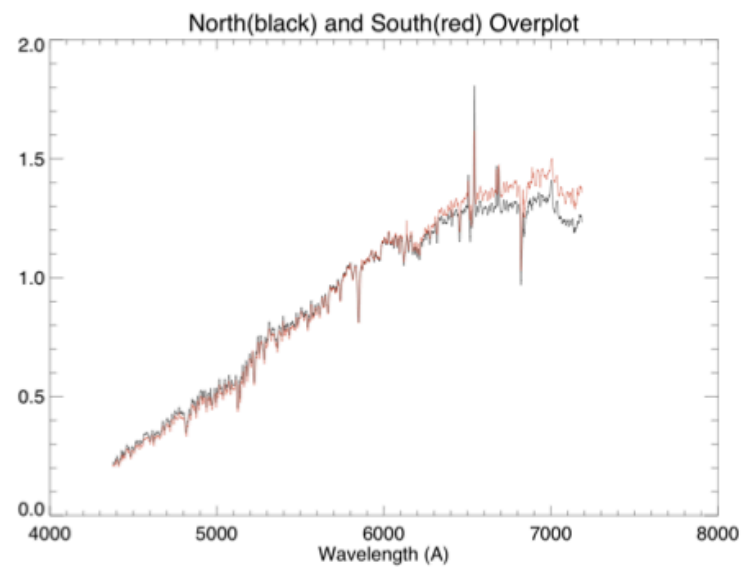

(a)

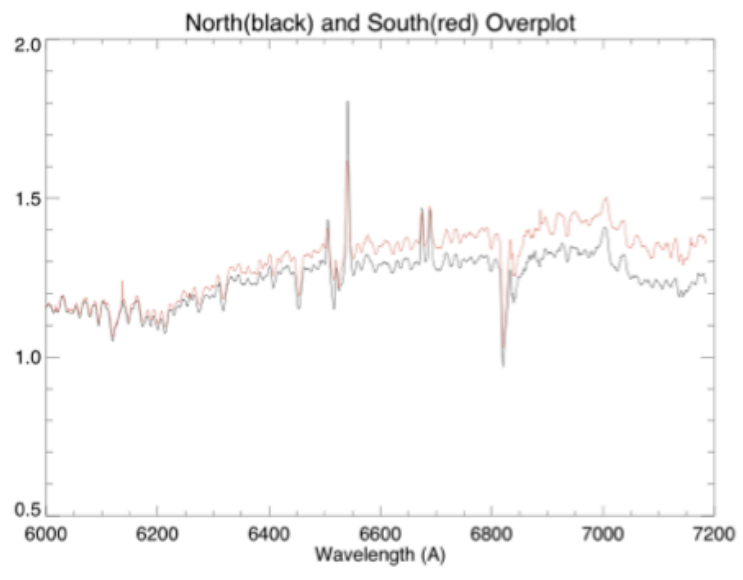

(b)

Figure 10: NGC 4736 overplot of 2.5" South and 2.5" North. The unknown source, plotted in black, is bluer than the local environment, or baseline, plotted in red.

4. If we look at the unknown source, primary, and baseline, we can remove the effects of the local environment by subtracting the baseline. This process removes all gas and dust that is between the observer and the sources. The resulting spectra are representative of characteristics of just the sources (Figure 11).

5. High excitation lines are stronger at the unknown source compared to the baseline at 2.5 " south of the primary source. Figure $11 \mathrm{~b}$ reveals the excess emission in the north in comparison to the baseline, or local environment of the galaxy nucleus. 


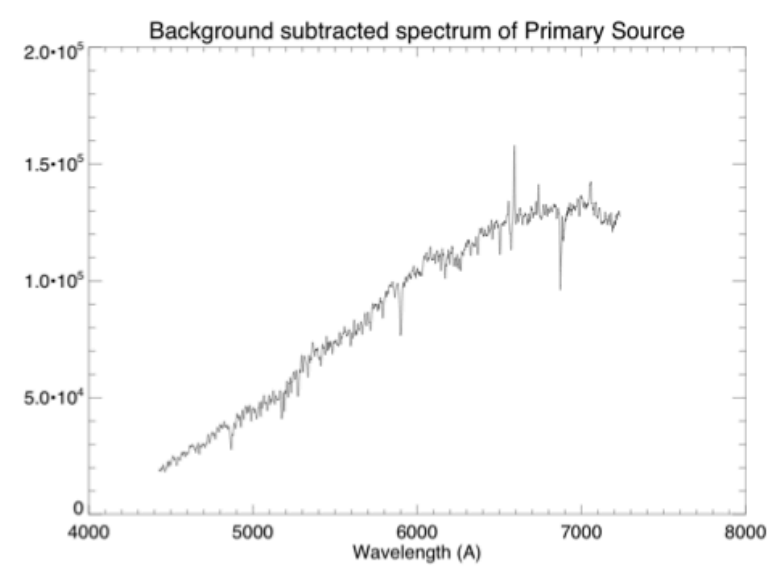

(a)

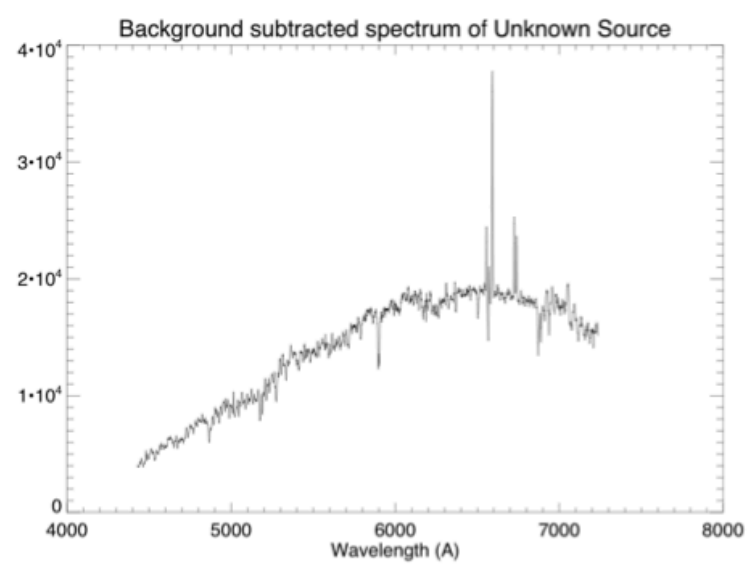

(b)

Figure 11: Spectra of the baseline-subtracted unknown source (left) and primary source (right). With the removed baseline, weaker lines like [OIII] are more prevalent.

6. Line strengths were calculated for the AGN diagnostic lines of the BPT-NII diagram to categorize the unknown source. Table 3 shows the best estimated line ratios of $[\mathrm{NII}] /[\mathrm{H \alpha}]$ and $[\mathrm{OIII}] /[\mathrm{H} \beta]$. We see very weak [OIII] emission in both the primary source and the unknown source (Figure 12).

Table 3: NGC 4736 ratio of $[\mathrm{NII}] /[\mathrm{H} \alpha]$ using the background subtracted spectra.

\begin{tabular}{ccccccc}
\hline \hline Location & {$[\mathrm{NII}]$} & {$[\mathrm{H} \alpha]$} & {$[\mathrm{OIII}]$} & {$[\mathrm{H} \beta]$} & $\log ([\mathrm{NII}] /[\mathrm{H} \alpha])$ & $\log ([\mathrm{OIII}] /[\mathrm{H} \beta])$ \\
\hline Unknown Source & 112000 & 4810 & 6040 & 28400 & 1.37 & -0.6 \\
Primary Source & 256000 & 9140 & 8160 & 155000 & 1.45 & -1.3 \\
\hline
\end{tabular}

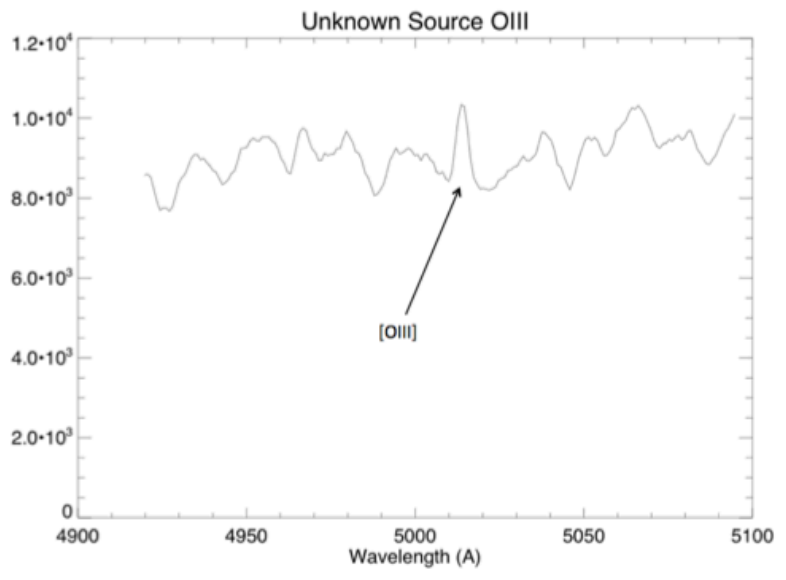

(a)

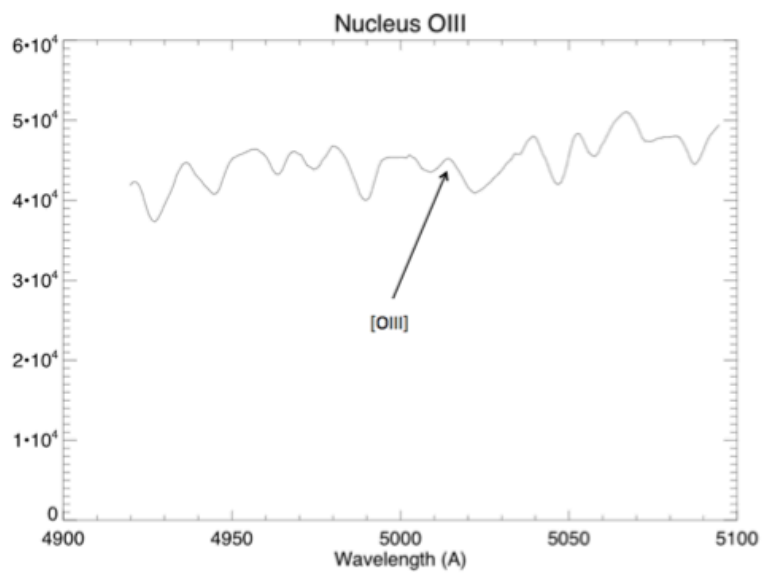

(b)

Figure 12: [OIII] (5007 $\AA$ ) zoomed in for the background-subtracted unknown source (left) and primary source (right). The spectra reveal very week [OIII] emission. 
To provide more evidence that the calculated ratios of $[\mathrm{NII}] /[\mathrm{H \alpha}]$ and $[\mathrm{OIII}] /[\mathrm{H} \beta]$ are in fact correct, we shifted our range for the line integral one pixel (0.1454") in each direction. We saw a vast increase in the value of the $[\mathrm{OIII}] /[\mathrm{H} \beta]$ ratio, and this was our largest source of uncertainty. In this system, [OIII] is such a weak line in comparison to [H $\beta$, our calculated line strength was very dependent on the line width and placement of the baseline. With a one-pixel shift, we calculated an upper limit of 0.6 for the ratio of $[\mathrm{OIII}] /[\mathrm{H} \beta]$. As a result, we placed the primary and secondary sources on the BPT-NII diagram (Figure 13).

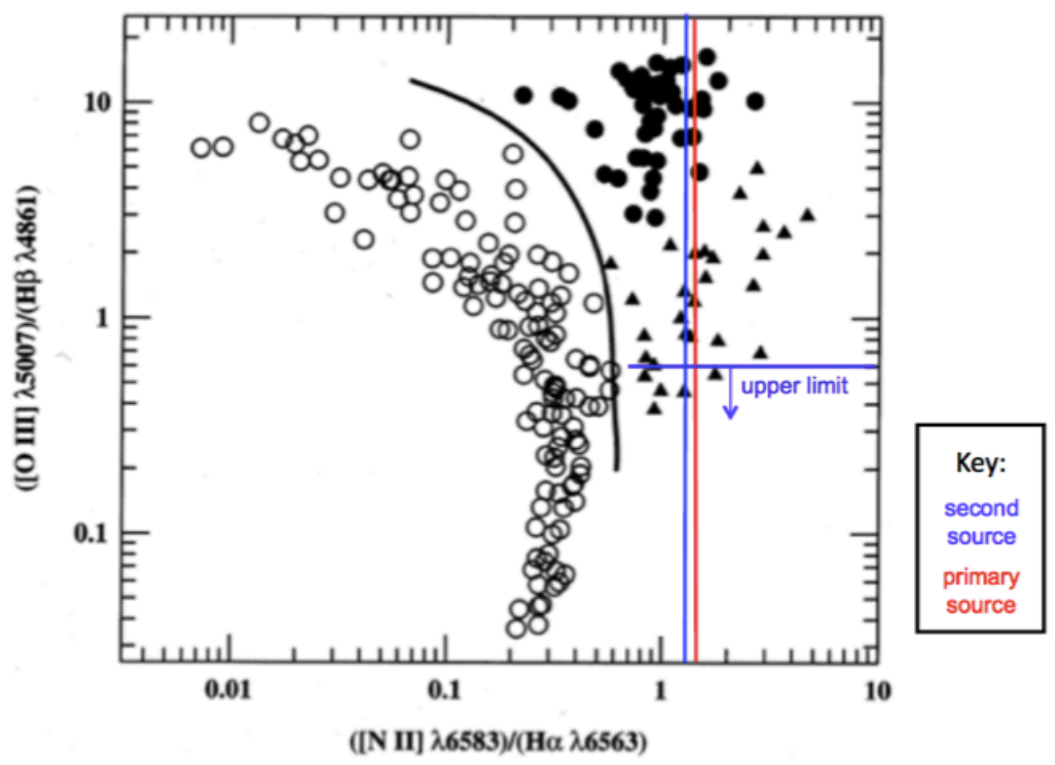

Figure 13: BPT-NII diagram with both nuclear sources plotted using diagnostic line ratios where the local environment has been removed. Only an upper bound can be placed on the $[\mathrm{OIII}] /[\mathrm{H} \alpha]$ due to the weak emission of $[\mathrm{OIII}]$.

\section{CONCLUSIONS}

The second unknown source in the nucleus of NGC 4736 has been observed in multiple wavelengths, and yet, no conclusions have been drawn on its nature. The unknown source is luminous as it emits in the x-ray and visible light, exciting the material around it. This leads us to question whether the second source is luminous enough to be a low luminosity black hole.

Given that the primary source is in fact a LINER, we are led to conclude that the unknown second source must be also be a LINER as it falls very near the primary source on the BPT diagram. We are confident in our calculation of the $[\mathrm{NII}] /[\mathrm{H \alpha}]$ ratio, or the vertical placement, but can only place a vertical upper limit on our calculation of [OIII]/[H $\beta]$ due to the uncertainty in the line strength value of [OIII]. The upper limit falls in the realm of the LINERs, supporting our conclusion.

In the future we plan to reduce and analyze existing infrared data to back up our initial conclusion. If the infrared data confirms our conclusion, we will be able to confidently say that our second source is in fact the third, and closest, dual AGN system to Earth. With the system in 
late stages of merging, astronomers will be able to study the system in great detail to refine merging models and test theories that rely on the existence of these systems.

\section{APPENDIX}

\section{A. RACHEL MASON, OFFICIAL PROPOSAL}

\section{Science Justification:}

Binary black holes (BBH) are expected on theoretical grounds to be common in galaxy nuclei, and are invoked to explain a range of phenomena. If two merging galaxies harbour supermassive black holes, then the black holes will themselves form a binary system during the course of the merger and eventually coalesce, although the timescales for and mechanisms in operation during this process are the subject of some debate. The resulting BBH have been suggested to be triggers of star formation (Taniguchi \& Wada 1996), to initiate the formation of dusty AGN tori (Zier \& Biermann 2001,2002), and may explain observations as diverse as helically-distorted radio jets (e.g. Tateyama et al. 1998) and quasi-periodic variations in the light curves of some BL Lac objects (e.g. Pursimo et al. 2000). Despite the theoretical predictions and their potential importance to astrophysics, though, evidence for the existence of BBH remains fairly sparse.

Several examples exist of images of quasars with another quasar nearby and at the same redshift. Although most of these are the result of gravitational lensing, a handful - based on spectral differences between the two components - seem to be genuinely physically associated quasar pairs, with the smallest separation being $2.5 \mathrm{kpc}$. At closer separations, nearby galaxies in the late stages of a merger are the obvious systems in which to search for BBH, and many of these do in fact have double optical or IR nuclei. However, it is only in rare cases that both nuclei exhibit AGN activity, that is, definitely harbour supermassive black holes. The first to be discovered was NGC 6240, in which Chandra imaging revealed two flat-spectrum, heavily absorbed X-ray sources at a projected separation of 1.5” (1.4 kpc; Komossa et al., 2003). The second, and so far final, candidate is Arp 299 (Ballo et al. 2004).

The recent discovery by Maoz et al. (2005) of variability in a pair of UV sources in the nucleus of the nearby LINER, NGC 4736, may have changed this. Morphological peculiarities in this galaxy led Maoz et al. $(1995,1996)$ to speculate that it is in the final stages of a merger, and the significant variability of the nuclear sources, which are of comparable brightness, is likely to indicate a significant nonstellar contribution to the UV fluxes of both. At a separation of only 60 pc (2.5" at $5 \mathrm{Mpc}$ ), this system would, if confirmed as a $\mathrm{BBH}$, represent a much more advanced stage of merging than the previous examples and be a significant step in validating models of galaxy mergers as well as providing a prime opportunity to study a nearby system in detail. The process(es) giving rise to the off-nuclear UV emission ("NGC 4736b"), however, are not at all well constrained.

To clarify the nature of the nuclear UV sources in NGC 4736, we propose GMOS-N long-slit spectroscopy of this enigmatic LINER. The variability of the sources rules out a stellar cluster as 
the sole source of their UV emission, but it is possible that a single W-R or LBV star could dominate their light output. The "nuclear" UV source (spatially coincident with the radio, optical and IR core) contains a variable hard X-ray point source with a spectral slope typical of those observed in Seyfert nuclei (Roberts et al. 1999, Eracleous et al. 2002), and does not show the spectral features characteristic of luminous, evolved stars (Cid Fernandes et al. 2004), strongly suggesting an AGN origin for the bulk of its flux. Whether an evolved high-mass star could account for NGC 4736b is easily testable from an optical spectrum, for example by searching for strong HeII 4686 line emission.

As the nuclear point source has properties consistent with its hosting an AGN, jet activity, (synchrotron radiation or gas heated by beamed radiation from the nucleus) could account for the UV emission from NGC 4736b, whose spectral properties are completely unknown. The nondetection of radio emission from NGC 4736b (Kording et al. 2005) suggests that the synchrotron explanation is unlikely, but we can test whether it is simply shocked gas by examining the continuum along the slit: enhanced line emission (possibly including high-excitation species) would be expected from shocked gas.

Finally, NGC 4736b could itself be a low-luminosity AGN. Following Ho et al. (1997), optical line ratios will be used to classify that source as a Seyfert, LINER, HII region, or transition object. This classification scheme does not necessarily unambiguously identify AGN but if the alternative explanations can be discounted, detection of a LINER-like spectrum combined with UV variability would itself be very suggestive that this galaxy does indeed harbour the closest known interacting supermassive black holes. NGC 4736 is a bright, nearby object and useful data can be obtained in a short amount of time under poor weather conditions; this programme represents a very modest investment of telescope time for a potentially very exciting result.

\section{Technical Information:}

Optical spectra of NGC 4736 have been taken by Ho et al. 1995 using a 2" slit orientated such that NGC 4736b was not in the slit. Extracted across 4", the flux density in their spectrum was around $25-30 \times 10^{-15} \mathrm{erg} / \mathrm{sec} / \mathrm{cm}^{2 / A}$ from $4200 \AA-6800 \AA$. Under the probably conservative assumption of spatially uniform brightness, this gives a surface brightness of about $3 \times 10^{-15}$ $\mathrm{erg} / \mathrm{sec} / \mathrm{cm}^{2 / \mathrm{A}}$.

The flux of NGC 4736b is not known at optical wavelengths, but it has been up to $30 \%$ brighter and $70 \%$ fainter than the nucleus at UV (2500 $\AA$ and $3300 \AA)$ wavelengths and has a bluer colour (Maoz et al. 1995; 2005). We adopt a V-band surface brightness of $1{\mathrm{X} 10^{-15}}^{-15}$ $\mathrm{erg} / \mathrm{sec} / \mathrm{cm}^{2 / \mathrm{A}}$ per sq. arcsec on which to base our integration time estimates. As the contribution of the point source over the diffuse stellar emission and the strength of any lines from that source are unknown, our strategy will be to maximise the $\mathrm{S} / \mathrm{N}$ on the continuum for the best chance of detecting weak emission lines. To minimise dilution of the point source spectrum by stellar emission, we will use the 0.75 " slit combined with 70\%-ile image quality.

The most useful diagnostic emission lines are likely to be $\mathrm{H} \alpha(6563 \mathrm{~A}), \mathrm{H} \beta$ (4861 A), [O III] (5007 A), [O I] (6300 A), [N II] (6583 A), [S II] (6716 A), (6731 A), [HeII] (4686 A) and [He I] $(5876 \mathrm{~A})$. We therefore use the B600 grating (no filter) with central wavelength $\sim 580 \mathrm{~nm}$ for $\mathrm{R} \sim 800$, and 2x2 CCD binning. We use conditions of 70\%-ile IQ, 90\%-ile CC, SB=any, WV=any, 2 airmasses. This setup gives a S/N of approximately 50-90 in 0.75 " along the slit over the 
wavelength range of interest in $8 \times 30$ min exposures. This should be enough to give us a sporting chance of detecting emission lines from NGC $4736 \mathrm{~b}$.

The baseline calibrations will be sufficient, so allowing for acquisition, we request 5 hours for this project. Please note that the time calculation is for bright time; in grey or dark time we could complete the project in about 1.5 hours.

\section{B. SPECTROSCOPY}

In order to analyze what exactly is happening in these systems, we want to resolve and interpret the optical spectra of the nucleus of the galaxy. A spectrum is a collection of light from a single star, a whole galaxy, or any other astronomical object, which helps in determining physical properties. In practice, an instrument called a spectrometer is used to collect the light and digitize it onto a computer.

For my research, collecting a spectrum from NGC 4736 involves the use of an astronomical spectrometer, also known as a spectrograph. The basic function of a spectrometer (Figure 14) is to take in light and disperse it into its spectral components, digitize the signal as a function of pixels, and read it out through a computer. The first step in this process is to direct light through the telescope into the spectrometer through a narrow aperture known as an entrance slit. The divergent light is then collimated by a concave mirror and directed onto a grating. The grating disperses the spectral components of the light, which are then focused by a second concave mirror and directed onto the detector. Once the light is imaged onto the detector the data is digitized and read out to a computer. The pixel scale is later calibrated to wavelength, so the resulting output data is in the form of wavelength versus intensity. 


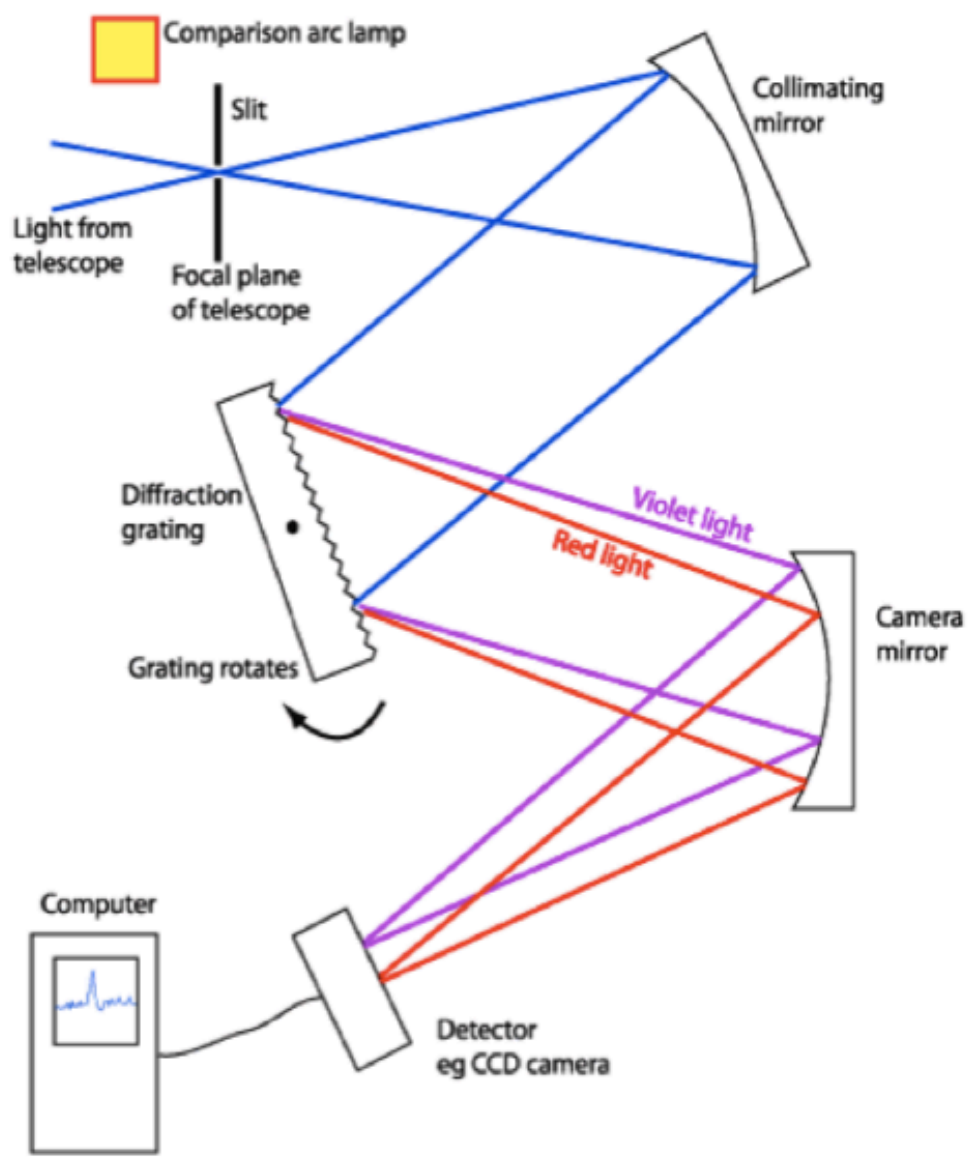

A Schematic Diagram of a Slit Spectrograph

Figure 14: The slit spectrograph diagrams the path the light takes through the telescope and onto the CCD detector where it is then read by the computer.

Because we analyze data from the spectrometer in the form of wavelength, it is important that we understand some properties of light to be able to read the accompanying spectra. In 1864 Scottish theoretical physicist James Maxwell described the precise relationship between electric fields and magnetic fields, thus showing that light was made of electromagnetic waves. For example, if a pebble is dropped over a body of water, then it creates waves, which move radially outward from where the pebble was dropped. These water waves contain both peaks and troughs with a given distance between each (Figure 15). For a steady wave, this quantity is consistent and we call it the wavelength of the wave, denoted by the Greek letter lambda $(\lambda)$. 


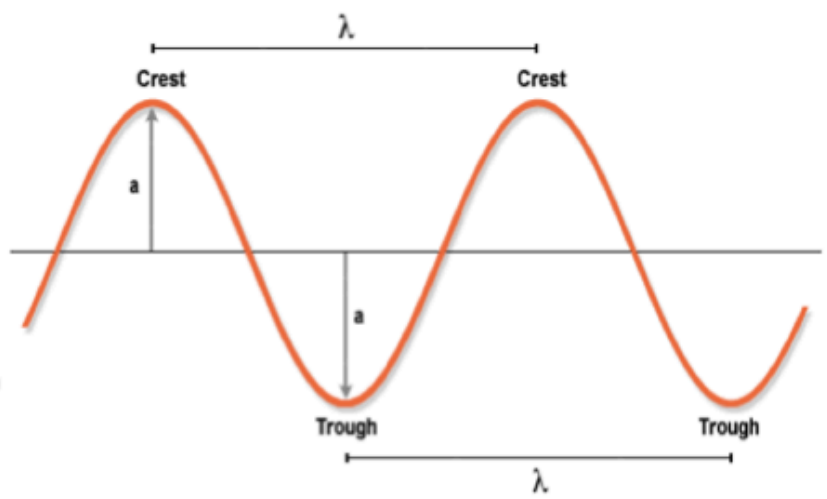

Figure 15: The wave properties of light give a distance between toughs or crests, defined as lambda $(\lambda)$, which defines where the light is on the electromagnetic spectrum.

All light has a wavelength, and that wavelength characterizes where it lies on the electromagnetic spectrum. Typically, when we think of light, we think of what we see, visible light. However, this is only a small portion of all of light, or what scientists call the electromagnetic spectrum (Figure 16). The electromagnetic spectrum ranges from gamma rays, which have the shortest wavelength all the way up to radio waves, which have the longest wavelength. Visible light, also known as optical light, falls on the spectrum between ultraviolet (UV) and infrared (IR). Looking at astronomical objects with different types of electromagnetic radiation is extremely important because it enhances or decreases what can be seen. For example, there is a lot of dust in space, which creates a barrier, limiting the distance to which objects can be viewed in the optical. However, IR radiation can more easily travel through dusty regions of space and therefore we can observe objects in highly obscured region. As a result, different types of electromagnetic radiation give us different windows to view the universe.

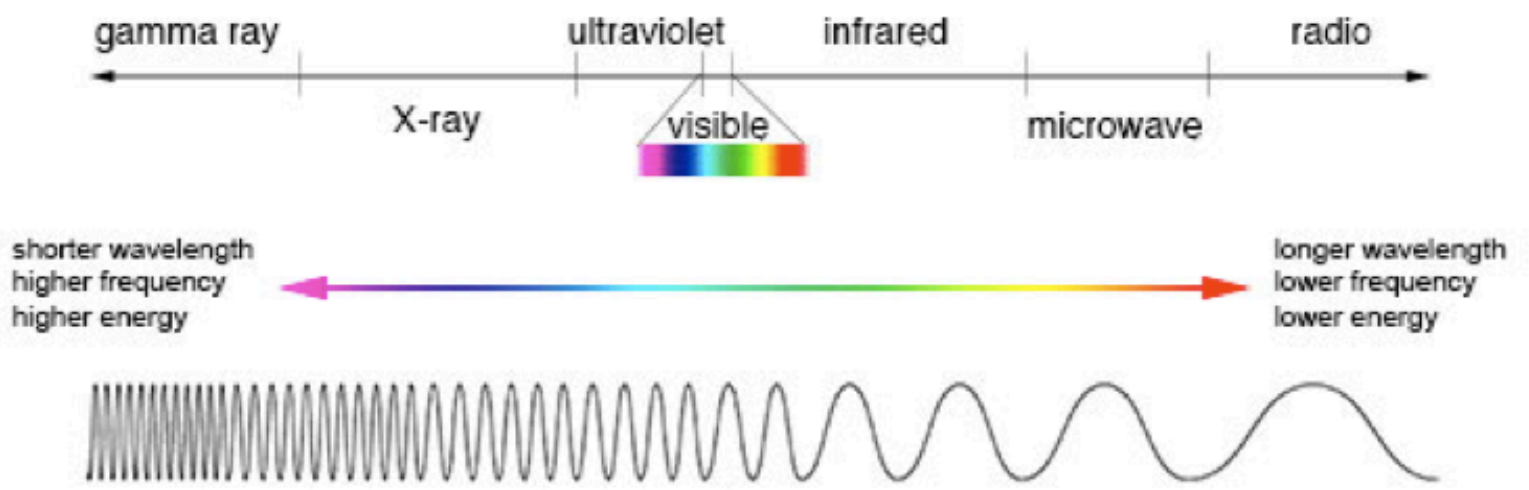

Figure 16: The Electromagnetic Spectrum displays the range of all light in the universe from high energy, short waves to lower energy, longer waves. The visible light is only a small portion of the entire Electromagnetic Spectrum. 
For my project, NGC 4736 was observed in the X-Ray when the two sources were first discovered. While the second source was not seen in UV, we were still able to learn about its properties. My goal now is to analyze the second source in the optical wavelengths to continue narrowing down its properties, some of which will hopefully be easily detectable in the optical.

I expect to see two types of spectra in my data-absorption and emission spectra. I do not expect to see any continuum in the spectra data because the resolution of the spectrometer is high enough that every line in the data is absorption or emission. An emission spectrum involves bright lines associated with increased intensity while an absorption spectrum involves lack of intensity, or very negative peaks in the spectra. The emission lines correspond to photons of discrete energies that are emitted when excited atomic states in the gas make transitions back to lower-lying levels while absorption lines are caused by atoms in the gas that are absorbed at characteristic frequencies. These spectral lines are critical in analyzing the properties of the astronomical object because they allow us to determine the characteristics of the object we are interested in. The wavelengths at which we observe emission and absorption lines correlate with a specific element or compound that is being radiated by a luminous object. An example of a spectrum plot is shown below in Figure 17. The peaks of the plot are the emission lines, while the dips are the absorption lines.

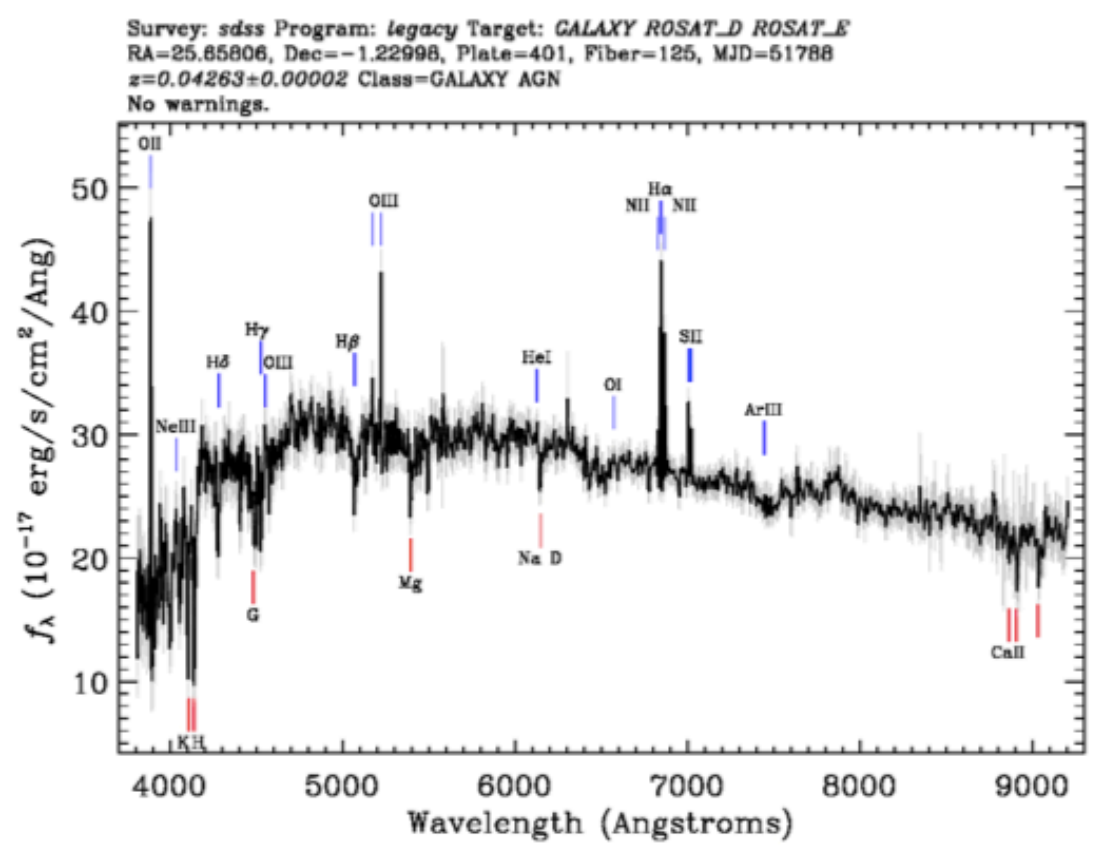

Figure 17: Example spectra containing absorption lines (red) and emission lines (blue).

\section{DATA REDUCTION}

The first step to the data reduction process was to inspect all images to pull out the saturated or irregular files. The data files were also placed into groups based on their file type so that all operations could be performed simultaneously on the data type. 


\section{1 CREATE MASTER BIAS}

As part of the data package, we received an already compiled master bias file. The preprocessed master bias file was created from many biases that are not overscan corrected but are trimmed, and finally averaged together.

GBIAS created a combined GMOS bias image from raw bias images. The raw bias images from GMOS had exposure time 1 sec. These images are taken with the GMOS shutter closed. The input images can be raw or prepared with GPREPARE. If they are raw, GBIAS will call GPREPARE first. The images were trimmed and the overscan level was not subtracted off the input images. The bias images were then combined with the task GEMCOMBINE. Lastly, they were averaged using the median combined option.

\section{C.2 NORMALIZE THE FLAT FIELD}

A similar process was done to create the normalized flat field. The flats were not overscan corrected, but were trimmed. They were also bias subtracted. The response of the flat source was fit detector by detector instead of slit by slit. Each row was then divided by its corresponding fit. The main function of GSFLAT is to remove the GCAL+GMOS spectral response and the GCAL uneven illumination from the flat-field image and leave only the pixel-to-pixel variations and the fringing.

GSFLAT is used to generate a normalized GCAL spectroscopic flat field. GSFLAT accepts as input either a single flat field image or a list of images, which are combined using IMCOMBINE before the response function is determined. The input flats were raw, in which case GPREPARE and GSREDUCE were called. GSREDUCE multiplies each extension by the gain using GGAIN. GMOSAIC was called to mosaic the science extensions into a single frame. The slits are identified using GSCUT.

After processing with GSREDUCE, and GSCUT, GSFLAT fits the response function with a single continuum fit made to the average of the rows within each detector and all the rows are normalized using that fit, retaining any spatial structure. For long slit data, only $85 \%$ of the central third of the slit length is used in determining the normalization, to preserve the flux at the middle and avoid errors due to the bridges.

\section{C.3 REDUCE THE OBJECT DATA}

GSREDUCE is used for basic reductions of GMOS longslit and MOS spectroscopic images. The reduction steps are defined by the flags $\mathrm{fl}_{\text {over }}, \mathrm{fl}_{\text {trim }}, \mathrm{fl}_{\text {bias }}, \mathrm{fl}_{\mathrm{gscrrej}}, \mathrm{fl}_{\text {dark }}, \mathrm{fl} \mathrm{f}_{\text {flat }}, \mathrm{fl}_{\mathrm{gmosai}}, \mathrm{fl} \mathrm{f}_{\mathrm{fixpix}}$, $\mathrm{fl}_{\text {gsappwave, }} \mathrm{fl}_{\text {cut }}$, and $\mathrm{fl}_{\text {title. }}$. For our data reduction we had the overscan subtraction off, trimming on, flat field correcting on, mosaic on, and wavelength solution on.

\section{C.4 WAVELENGTH CALIBRATE}


The ARC data was again reduced in a similar fashion as above with the object data using GSREDUCE. Then, GSWAVELENGTH was used for establishing the wavelength calibration for the GMOS spectra. We performed our wavelength fitting without using the interact mode, which we had previously already done. We found our results using interact on and off to be consistent. GSTRANSFORM was applied to fit a 2D wavelength solution to the data.

\section{C.5 SKY SUBTRACT}

GSSKYSUB was used to subtract sky from the GMOS spectra. The resulting sky sample is usually of the form "r1:r2,r3:r4", where r1:r2 defines the rows on one side of the object aperture and r3:r4 defines the rows on the other side of the object aperture.

\section{ACKNOWLEDGEMENTS}

I would like to thank Gemini Observatory and Dr. Rachel Mason for supplying the data to our research group and Dr. Scott Fisher for acquiring the data and allowing his undergraduate students the opportunity to experience front line research. I would also like to thank Dr. Elsa Johnson and Dr. Joseph Fracchia for their support serving on my committee and Teiler Kwan and Jeremy Bullis for their assistance and support throughout the entire process. Lastly, I want to thank the Oregon NASA Space Grant Consortium for their support in funding my research. This project would not have been possible without all of the support I received.

\section{REFERENCES}

Abramowicz, M., Gunnlaugur Bjornsson, and J. E. Pringle. Theory of Black Hole Accretion Disks. Cambridge, UK: Cambridge UP, 1998. Print.

Alloin, D. M., Rachel Johnson, and Paulina Lira. Physics of Active Galactic Nuclei at All Scales. Berlin: Springer, 2006. Print.

Ball, David W. The Basics of Spectroscopy. Bellingham, WA: SPIE-The International Society for Optical Engineering, 2001. Print.

Beckmann, Volker, and Chris R. Shrader. Active Galactic Nuclei. Weinheim, Germany: Wiley$\mathrm{VCH}$, 2012. Print.

Begelman, Mitchell C., and Martin J. Rees. Gravity's Fatal Attraction: Black Holes in the Universe. New York: Scientific American Library, 1996. Print.

Constantin, Anca, and Anil C. Seth. "M94 As a Unique Testbed for Black Hole Mass Estimates and AGN Activity at Low Luminosities.” Advances in Astronomy 11th ser. 5.2 (2011) Print.

Cui, W., D. Feldkhun, and R. Braun. "Detection of Compact Nuclear X-Ray Emission in NGC 4736." The Astrophysical Journal 477 (1997): 693-99. Print. 
Eracleous, Michael, Joseph C. Shields, George Chartas, and Edward C. Moran. "Three LINERs Under the Chandra X-Ray Microscope.” The Astrophysical Journal 565 (2002): 108-24. IOP Science. Web. 2 Feb. 2014.

Frammert, Hartmut, and Kristine Kronberg. "Messier 94.” Messier Object 94. SEDS, 2 Sept. 2007. Web. 06 Feb. 2014.

Frolov, V. P., and Andrei Zelnikov. Introduction to Black Hole Physics. Oxford: Oxford UP, USA, 2011. Print.

“How Does a Spectrometer Work?” BW Tek. N.p., 2014. Web. 06 Feb. 2014.

Giacconi, Riccardo, Lex Kaper, HeUVel, Edward Peter Jacobus Van Den, and P. A. Woudt. Black Holes in Binaries and Galactic Nuclei: Diagnostics, Demography, and Formation: Proceedings of the ESO Workshop Held at Garching, Germany, 6-8 September 1999, in Honour of Riccardo Giacconi. Berlin: Springer, 2001. Print.

Howell, S.B. “CCD Imaging.” Handbook of CCD Astronomy. Cambridge, U.K.: Cambridge UP, 2000. 47-74. Print.

Jalocha, Joanna, Lukasz Bratek, and Marek Kutschera. "Is Dark Matter Present in NGC 4736? An Iterative Spectral Method for Finding Mass Distribution in Spiral Galaxies.” The Astrophysical Journal 679 (2008): 373-78. IOP Science. Web. 3 Feb. 2014.

Kaper, L., E.P.J. Van Den HeUVel, and P.A. Woudt. Black Holes in Binaries and Galactic Nuclei: Diagnostics, Demography, and Formation. New York: Springer, 2001. Print.

Kitchin, C. R. Galaxies in Turmoil: The Active and Starburst Galaxies and the Black Holes That Drive Them. London: Springer, 2007. Print.

Koekemoer, Anton, and Mario Livio, eds. Black Holes: Proceedings of the Space Telescope Science Institute Symposium, Held in Baltimore, Maryland, April 23-26, 2007. Cambridge, UK: Cambridge UP, 2011. Print.

Krolik, Julian Henry. Active Galactic Nuclei: From the Central Black Hole to the Galactic Environment. Princeton, NJ: Princeton UP, 1999. Print.

Lin, Dacheng, Jimmy A. irwin, Didier Barret, and Ronald A. Remillard. "Discovery of Highly Variable Dipping Ultraluminous X-Ray Source in M94.” Astrophysical Journal 11th ser. 5.2 (2013) Print.

Maoz, Dan, Neil M. Nagar, Heino Falcke, and Andrew S. Wilson. "The Murmur of the Sleeping Black Hole: Detection of Nuclear Ultraviolet Variability in LINER Galaxies." The Astrophysical Journal 625.2 (2005): 699-715. Print.

Mason, Rachel. "Proposal for NGC3736 GMOS-N Optical Spectroscopy.” Thesis. Gemini Observatory, 2007. HubbleSite. NSF. 
Massey, P. “A User's Guide to CCD Reductions with IRAF.” IRAF NOAO., 15 Feb. 1997.

Miller, Joseph S. Astrophysics of Active Galaxies and Quasi-Stellar Objects. Mill Valley, CA: University Science, 1985. Print.

Osterbrock, Donald E. Astrophysics of Gaseous Nebulae and Active Galactic Nuclei. Mill Valley, CA: University Science, 1989. Print.

Peterson, Bradley M. An Introduction to Active Galactic Nuclei. Cambridge: Cambridge UP, 1997. Print.

Roberts, Mallory S. E., and Robert W. Romani. "The ASCA Catalog of Potential X-Ray Counterparts of GeV Sources.” The Astrophysical Journal Supplement Series 122 (2001): 451-65. IOP Science. Web. 2 Feb. 2014.

Robinson, Keith. Spectroscopy: The Key to the Stars. New York: Springer, 2007. Print.

Steiner, J. E., R. B. Menezes, T. V. Ricci, and A. S. Oliveira. "PCA Tomography: How to Extract Information from Data Cubes.” Monthly Notices of the Royal Astronomical Society 395 (2009): 64-75. Print.

Villard, Ray, and John Kormendy. "Black Holes Shed Light on Galaxy Formation.” HubbleSite. HubbleSite, 5 June 2000. Web. 06 Feb. 2014. 